\title{
DIFFERENTIABLE PSEUDO-FREE CIRCLE ACTIONS ON HOMOTOPY SPHERES ${ }^{1}$
}

\author{
CHAO-CHU LIANG
}

\begin{abstract}
Let $G$ denote the circle group, $\varphi$ a differentiable pseudo-free $G$-action of type $\left(p_{1}, \ldots, p_{k}\right)$ on a homotopy sphere $\Sigma^{2 n+1}$, and $X$ the vector field induced by $\varphi$. If $w$ is a $G$-invariant 1 -form satisfying $w(X)=1$, then we will prove that $\int_{\Sigma} w \wedge(d w)^{n}= \pm\left(p_{1} p_{2} \cdots p_{k}\right)^{-1}$.
\end{abstract}

Let $R$ denote the set of real numbers, $Z$ the set of integers, and $G=$ $R / Z$-the circle group.

Suppose we have an effective $G$-action without fixed point on a smooth compact manifold $M^{2 n+1}$. This action induces a vector field $X$ of period 1 on $M$. Let $p$ denote the least common multiple of the orders of the isotropy groups of the elements of $M$. If $w$ is a $G$-invariant 1-form such that $w(X)=1$, then Weinstein [9] showed that $p^{n} \int_{M} w \wedge(d w)^{n}$ is an integer. The aim of this paper is to compute Weinstein's integers in certain cases.

An effective $G$-action $\varphi$ on a homotopy sphere $\Sigma^{2 n+1}$ is called pseudo-free of type $\left(p_{1}, p_{2}, \ldots, p_{k}\right)$, if there exist $k$ isolated exceptional orbits such that $Z / p_{i} Z$ (the cyclic group of order $p_{i}$ ) is the isotropy group for the points on the $i$ th orbit, and the integers $p_{1}, \ldots, p_{k}$ are relatively prime to one another, [5], [6]. In the following, we let $p=p_{1} p_{2} \cdots p_{k}$.

Let $S^{1}$ denote the group of complex numbers (under multiplication) of absolute value 1. Let $S^{2 n+1}$ be the unit sphere in the complex $(n+1)$-space $\mathrm{C}^{n+1}$. Then there exists a linear pseudo-free $S^{1}$-action $\psi_{p}$ on $S^{2 n+1}$, given by the equation

$$
g\left(z_{0}, z_{1}, \ldots, z_{n}\right)=\left(g^{p} z_{0}, g z_{1}, \ldots, g z_{n}\right) .
$$

In order to use the formula in [9], we construct another $G$-action $\varphi_{p}$, which is induced from $\psi_{p}$ by the map $G \rightarrow S^{1}$ sending $g$ to $e^{2 \pi i g}$.

Proposition. Let $X$ denote the vector field on $S=S^{2 n+1}$ induced by $\varphi_{p}$, and let $w$ be a G-invariant 1-form satisfying $w(X)=1$. Then we have $\int_{S} w \wedge(d w)^{n}$ $= \pm p^{-1}$.

Proof. The orbit space $S / G$ is a $V$-manifold, [9]. For $V$-manifolds, we have a theory of differential forms, fibre bundles etc., and the deRham theorem holds, [1], [7], [8]. Furthermore, there is a Chern-Weil theory of characteristic classes, [4], [9]. In particular, if $E$ is a principal circle bundle

Received by the editors November 30, 1977 and, in revised form, March 17, 1978.

AMS (MOS) subject classifications (1970). Primary 57E15, 57E25.

Key words and phrases. Differentiable pseudo-free actions, $V$-manifolds, characteristic classes.

${ }^{1}$ Supported by the University of Kansas General Research Fund.

() American Mathematical Society 1978 
over a $V$-manifold, then one can compute a form representing the first Chern class $c_{1}(E)$ in the usual way from a connection on $E$.

Let $K=Z / p Z \subseteq G$, then $S / K \rightarrow S / G$ is a principal $G / K$ bundle, and $S / K$ is a $V$-manifold. The $G$-invariant 1 -form $w$ induces a $G / K$-invariant 1 -form $\bar{w}$ on $S / K$. Also, $X$ induces a vector field $\bar{X}$ on $S / K$. The vector field $p^{-1} \bar{X}$ generates the $G / K$-action on $S / K$ (its flow has period 1 ), so $p \bar{w}$ is a connection form for the bundle $S / K \rightarrow S / G$. By the Chern-Weil theory, $c_{1}(S / K)$ is represented by $p \Omega_{G}$, where $\Omega_{G}$ on $S / G$ pulls back to $d \bar{w}$ (see [9, pp. 267-268], also cf. [2]).

It was shown in [3] that $S / G$ has the same integral cohomology as $\mathbf{C} P^{n}$, the complex projective space. Let $\beta_{1} \in H^{2}(S / G ; Z)=Z$ be a generator. It is easy to see that the Chern class of the principal bundle $S / K \rightarrow S / G$ is $\pm \beta_{1}$ (constructing a map from $S / K \rightarrow S / G$ to $S^{2 N+1} \rightarrow \mathbf{C P} P^{N}$ for some large $N$ by considering $\left.S^{2 n+1} / G=\left(S^{1} \times D^{2 n}\right) / G \cup\left(D^{2} \times S^{2 n-1}\right) / G\right)$. Thus the cohomology class $\left[\Omega_{G}\right]$ determined by $\Omega_{G}$ is equal to $\pm p^{-1} \beta_{1}$.

We have the following formula from [9, p. 268]:

$$
\int_{S} w \wedge(d w)^{n}=\int_{S / G} \Omega_{G}^{n}
$$

Let $\beta_{j} \in H^{2 j}(S / G ; Z)$ be the generator for $1 \leqslant j \leqslant n$. It is known that we may choose $\beta_{j}$ 's in such a way that $\beta_{1}^{2}=p \beta_{2}, \beta_{1}^{3}=p^{2} \beta_{3}, \ldots, \beta_{1}^{n}=p^{n-1} \beta_{n}$ [6, p. 95]. We also use $\beta_{k}$ to denote a $2 k$-form on $S / K$ representing $\beta_{k}$. Then we have $\left[\Omega_{G}\right]^{n}= \pm p^{-n} \beta_{1}^{n}= \pm p^{-1} \beta_{n}$, and $\int \Omega_{G}^{n}= \pm p^{-1} \int \beta_{n}= \pm p^{-1}$. Q.E.D.

Suppose that $\varphi$ is a differentiable pseudo-free $G$-action of type $\left(p_{1}, p_{2}, \ldots, p_{k}\right)$ on a homotopy sphere $\Sigma^{2 n+1}$. Then the main theorem of [5] asserts the existence of a $G$-equivariant map $F$ of $\Sigma^{2 n+1}$ into $S^{2 n+1}$ (with the action $\varphi_{p}$ ) of degree \pm 1 , that is, we have the following diagram:

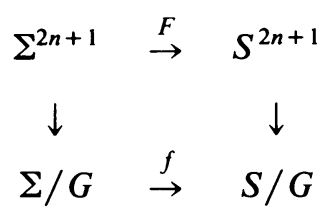

and the principal $G / K$-bundle $\Sigma / K \rightarrow \Sigma / G$ is induced by the homotopy equivalence $f: \Sigma / G \rightarrow S / G$. Therefore, $f^{*}: H^{*}(S / G ; Z) \rightarrow H^{*}(\Sigma / G ; Z)$ is a ring isomorphism, and we have $c_{1}(\Sigma / K)= \pm f^{*}\left(\beta_{1}\right)$. The proof of the proposition then yields:

THEOREM. Let $\varphi$ be a differentiable pseudo-free circle action of type $\left(p_{1}, \ldots, p_{k}\right)$ on a homotopy sphere $\Sigma^{2 n+1}$, and $X$ the vector field induced by $\varphi$. If $w$ is a $G$-invariant 1-form satisfying $w(X)=1$, then we have

$$
\int_{\Sigma} w \wedge(d w)^{n}= \pm p^{-1}, \quad \text { where } p=p_{1} p_{2} \cdots p_{k}
$$


The author wishes to thank the referee for pointing out several mistakes in the original manuscript.

\section{REFERENCES}

1. W. L. Baily, The decomposition theorem for $V$-manifolds, Amer. J. Math. 78 (1956), 862-888.

2. W. M. Boothby and H. C. Wang, On contact manifolds, Ann. of Math. 68 (1958), 721-734.

3. P. E. Conner and E. E. Floyd, Orbit spaces of circle groups of transformation, Ann. of Math. 67 (1958), 90-98.

4. S. Kobayashi and K. Nomizu, Foundations of differential geometry, Vol. II, Wiley, New York, 1969.

5. D. Montgomery and C. T. Yang, Homotopy equivalence and differentiable pseudo-free circle actions on homotopy spheres, Michigan Math. J. 20 (1973), 145-159.

6. Differentiable pseudo-free circle actions on homotopy seven spheres (Proc. Second Conference Complex Transformation Groups, Amherst, 1971), Lecture Notes in Math., vol. 298, Springer-Verlag, Berlin and New York, 1972, pp. 41-101.

7. I. Satake, On a generalization of the notion of manifold, Proc. Nat. Acad. Sci. U.S.A. 42 (1956), 359-365.

8. __ The Gauss-Bonnet theorem for V-manifolds, J. Math. Soc. Japan 9 (1957), $464-492$.

9. A. Weinstein, Symplectic $V$-manifolds, periodic orbits of Hamiltonian systems, and the volume of certain Riemannian manifolds, Comm. Pure Appl. Math. 30 (1977), 265-271.

Department of Mathematics, University of Kansas, LaWrence, Kansas 66045 